\title{
Acute Reversal of Experimental Diabetic Ketoacidosis in the Rat with (+)-Decanoylcarnitine
}

\author{
J. Denis McGarry and Daniel W. Foster \\ From the Departments of Internal Medicine and Biochemistry, The University \\ of Texas Southwestern Medical School at Dallas, Dallas, Texas 75235
}

A в S T R A C T The effect of (t)-decanoylcarnitine, a potent inhibitor of long-chain acylcarnitine transferase, was tested for its ability to inhibit hepatic ketogenesis both in the isolated perfused liver and in vivo in severely ketotic alloxan diabetic rats. In vitro the inhibitor caused an almost complete block in ketone body production. In vivo $(+)$-decanoylcarnitine caused a rapid reversal of ketosis under conditions where large doses of insulin had little effect. A combination of the two agents produced an even more striking fall in plasma ketone levels.

While (t)-decanoylcarnitine alone had no effect on plasma glucose levels it enhanced the hypoglycemic effect of insulin in anesthetized animals. Loss of this effect was noted in nonanesthetized animals, possibly as a result of increased muscle activity.

Studies in the isolated perfused liver indicated that the blockade of fatty acid oxidation and ketogenesis procluced by $(+)$-decanoylcarnitine was rapidly reversible upon removal of the inhibitor.

\section{INTRODUCTION}

Recent studies from this laboratory have been concerned with the mechanisms by which ketogenesis is regulated (1-6). It now seems clear that maximal ketogenesis requires that two conditions be fulfilled: (a) There must be an adequate delivery of the basic ketogenic substrate, fatty acid, to the liver, and (b) There must be a change in the metabolic "set" of the liver such that a greater fraction of the incoming fatty acids is shunted into the oxidative sequence relative to that utilized for triglyceride and phospholipid synthesis.

Failure to meet either requirement prevents the onset of ketone body overproduction or causes a reversion of

Dr. Foster is Research Career Development Awardee 5-AM9968, U. S. Public Health Service.

Received for publication 10 October 1972 and in revised form 17 November 1972. established ketosis to normal. Current understanding of the nature of the metabolic changes occurring in the hepatic cell during diabetic ketoacidosis is summarized in Fig. 1. Fatty acids extracted from the plasma by the liver can be metabolized by only two major pathways - they may be esterified to form triglycerides and phospholipids or they may enter the $\beta$-oxidation process. For reasons outlined in detail elsewhere $(1,6)$ it seems likely that the capacity for $\beta$-oxidation in the liver is fixed and high and that flux of fatty acyl carbon into this pathway (and ultimately the distribution of fatty acyl-CoA between esterification and the oxidative sequence) is determined by the rate of entry of fatty acyl$\mathrm{CoA}$ into the mitochondrion. As indicated, the enzyme catalyzing the translocation of the fatty acid molecule across the mitochondrial membrane is the long-chain fatty acylcarnitine transferase $(7,8)$. It is assumed (Fig. $1 A$ ) that the activity of this enzyme is inhibited in the normal fed state such that fatty acids are utilized by the liver primarily for the synthesis of triglyceride but that in fasting and diabetes (Fig. $1 B$ ) activation of the enzyme occurs with the consequence that the barrier to transport of the fatty acids into the mitochondrion is removed and the increased fatty acid load delivered to the liver is preferentially oxidized to acetoacetic and $\beta$-hydroxybutyric acids. In this regard it is of interest to note that Norum (9) has found increased activity of long-chain acylcarnitine transferase in liver homogenates and mitochondria from fasted and diabetic animals.

Should this formulation be correct, it follows that inhibition of the acylcarnitine transferase reaction would result in a blockade of fatty acid oxidation and an inhibition of ketogenesis. A specific inhibitor of the reaction, ( $(+)$-decanoylcarnitine, has been known for some time (10) and has been shown to block ketogenesis in isolated perfused livers taken from fasted animals (10-12). Moreover, when ketogenesis in livers from fasted rats was interrupted by $(+)$-decanoylcarni- 

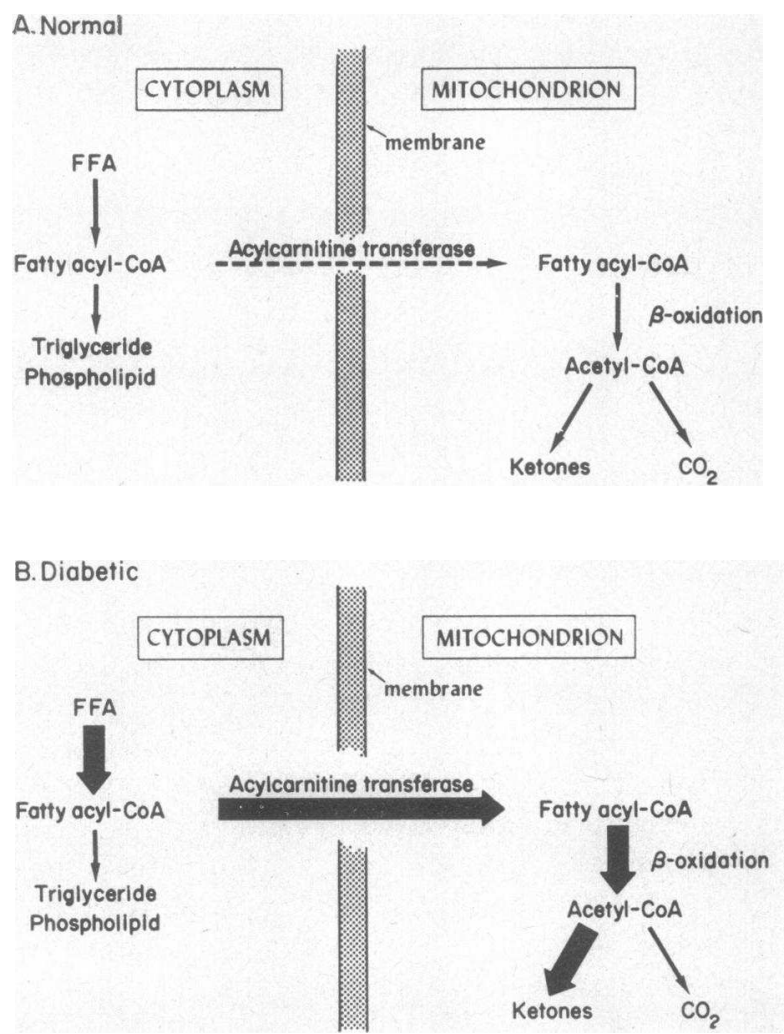

FIgURE 1 A possible model for the regulation of ketogenesis in diabetic acidosis. The pathways shown are highly simplified and schematized. Panel A indicates that in the normal state free fatty acid delivery to the liver is low and fatty acid oxidation is inhibited at the level of the acylcarnitine transferase reaction. Fatty acids are therefore utilized primarily for triglyceride synthesis. In panel B free fatty acid delivery is increased and the fatty acids are oxidized to ketone bodies through activation of the acylcarnitine transferase step. For a more detailed discussion of this formulation see references $1,6,7$.

tine, the perfused fatty acids were immediately shunted into hepatic triglycerides, restoring the pattern of fatty acid metabolism to that seen in normal liver (6).

For these reasons we decided to try $(+)$-decanoylcarnitine in the treatment of acute diabetic ketoacidosis in the rat. The results of the experiments to be presented indicate that $(+)$-decanoylcarnitine is a potent inhibitor of ketogenesis produced by uncontrolled diabetes both in vitro and in vivo. Moreover, when given in combination with insulin, (+)-decanoylcarnitine significantly reduces recovery times from diabetic acidosis and is much more effective than the hormone given alone.

\section{METHODS}

\section{Treatment of animals}

Male Sprague-Dawley rats weighing approximately $130 \mathrm{~g}$ and maintained on a diet containing $58.5 \%$ sucrose, $21 \%$ casein, and less than $1 \%$ fat (General Biochemicals Div., Mogul Corp., Chagrin Falls, Ohio) were used in all experiments. Severe diabetic ketoacidosis was induced by the intravenous injection of alloxan monohydrate at a dose of 70 $\mathrm{mg} / \mathrm{kg}$ body wt. Experiments were carried out $48 \mathrm{~h}$ after administration of the drug at which time body weight was approximately $100 \mathrm{~g}$. Only those animals with initial plasma ketones in excess of $1,000 \mu \mathrm{mol} / 100 \mathrm{ml}$ have been included in the studies reported below.

\section{In vitro studies-liver perfusion}

Recirculating perfusions. The effect of (+)-decanoylcarnitine on rates of ketogenesis was first studied in livers from acutely ketotic diabetic rats perfused with recirculating medium using the apparatus and techniques described elsewhere $(3,4)$. The perfusion fluid $(80 \mathrm{ml})$ consisted of washed, aged human erythrocytes suspended to an hematocrit of $20 \%$ in $5 \%$ bovine albumin dissolved in Krebs' bicarbonate buffer, $\mathrm{pH}$ 7.4. Sufficient oleic acid was included in the initial pool to give a fatty acid concentration in the cell-free fluid of approximately $0.7 \mathrm{mM}$. This concentration was maintained throughout the experimental period by the constant infusion of a solution containing $12.5 \mathrm{mM}$ oleate and $10 \%$ albumin dissolved in $0.9 \%$ sodium chloride, $\mathrm{pH} 7.4$, at a rate of $75 \mu \mathrm{l} / \mathrm{min}(6)$.

Nonrecirculating perfusions. In order to measure the rapidity with which hepatic ketogenesis is blocked by $(+)$ decanoylcarnitine and the time required for the recovery of the liver from the effects of the inhibitor it was necessary to utilize a nonrecirculating perfusion system. The methodology employed here was essentially the same as that used for the more conventional recirculating technique with the exception that an additional large reservoir of perfusion fluid (about $1,500 \mathrm{ml}$ ) was required since the venous flow from the liver was not returned to the oxygenation chamber. Using this system it was possible to infuse $(+)$-decanoylcarnitine into the perfusion circuit at a point just prior to entry into the liver, thus allowing the time of exposure of the tissue to the inhibitor to be varied at will.

\section{In vivo studies}

In order to follow the time sequence of the reversal of diabetic ketoacidosis with insulin and $(+)$-decanoylcarnitine animals were anesthetized either with pentobarbital, or lightly with ether, and no. 10 polyethylene catheters were placed in the femoral artery on one side and the inferior vena cava through the femoral vein on the other (13). The animals were then placed in individual restraining cages. When pentobarbital was used as anesthetic, experiments were started with the animals still asleep. Rats anesthetized with ether had recovered from anesthesia for at least 30 min before experiments were begun.

Samples of arterial blood $(0.1 \mathrm{ml})$ were collected into iced heparinized polyethylene tubes throughout the experiment for determination of plasma ketone and glucose concentrations (2). Heparin, when used, was given intravenously in a dose of 100 units prior to collection of the first sample. An infusion of $5 \%$ bovine albumin in $0.9 \%$ sodium chloride, $\mathrm{pH} 7.4$, containing insulin, $(+)$-decanoylcarnitine or a mixture of both agents was then given through the venous catheter as described in the legends to the figures. The amount of insulin given was sufficient to produce maximum effects as determined in preliminary experiments. Because of the small volumes of blood taken it was not possible to determine plasma FFA levels at each time point. 
For such measurements, a 2-3 $\mathrm{ml}$ specimen of blood was rapidly removed from the abdominal aorta at the conclusion of the experiment and compared with values obtained from control animals.

In experiments designed to determine the effects of a single injection of insulin and/or (+)-decanoylcarnitine rats that had not been catheterized or anesthetized were given, intravenously, $0.5 \mathrm{ml}$ of the albumin solutions containing insulin in the presence and absence of $(+)$-decanoylcarnitine as described above. The animals were then placed in conventional cages, and $2 \mathrm{~h}$ later they were anesthetized with pentobarbital immediately prior to collection of blood from the aorta.

\section{Analyses on blood and perfusion fluid}

All samples of blood and perfusion medium were centrifuged, and analyses were carried out on the erythrocyte-free supernatant fluid. Acetoacetate and $\beta$-hydroxybutyrate were determined enzymatically as described previously (2), and in the text the term "ketone bodies" will always refer to the sum of these two components. Glucose was measured using the glucose oxidase method (14), and free fatty acid levels were assayed by the method of Dole (15) as modified by Trout, Estes, and Friedberg (16).

\section{Materials}

Bovine albumin, Fraction V, was from Armour Pharmaceutical Co., Chicago, Ill. In all experiments with oleic acid and $(+)$-decanoylcarnitine these reagents were bound to the albumin and neutralized to $\mathrm{pH} 7.4$ before use. Glucagon-free insulin was a gift from Eli Lilly \& Co. and (+)decanoylcarnitine was generously provided by Dr. Y. Kawashima of the Otsuka Pharmaceutical Factory, Naruto, Tokushima, Japan. Oleic acid was purchased from ICN Nutritional Biochemicals, Inc., Cleveland, Ohio. All other reagents were obtained from commercial sources and were of the highest available grade.

\section{RESULTS}

Inhibition of ketogenesis in the diabetic liver in vitro. As noted above, $(+)$-decanoylcarnitine had been previously shown to have the capacity to inhibit ketogenesis in livers from fasted rats $(6,10-12)$. Ketone production by isolated perfused livers from fasted animals could also be partially inhibited with certain other antiketogenic agents such as lactate and glycerol $(4,17,18)$. Livers from severely ketotic diabetic rats, however, appear to be resistant to the effect of the latter compounds (3). For this reason we first examined the effect of $(+)$-decanoylcarnitine on acetoacetate and $\beta$ hydroxybutyrate production in the perfused liver from rats with acute diabetic ketoacidosis. The results are shown in Fig. 2 where it can be seen that ketone body synthesis was essentially completely inhibited after 15 min of perfusion with the nonphysiological carnitine ester.

Reversal of diabetic ketoacidosis with (+)-decanoylcarnitine and insulin-anesthetized animals. In the first set of in vivo experiments rats were anesthetized with pentobarbital for implantation of catheters, and studies

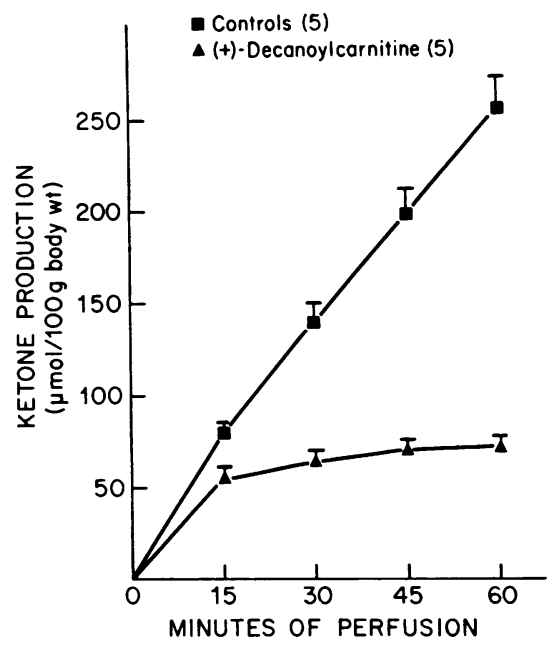

FIgURE 2 The effect of $(+)$-decanoylcarnitine on ketogenesis in perfused livers from alloxan diabetic rats. Livers were perfused with oleic acid $(0.7 \mathrm{mM})$ in the presence and absence of $(+)$-decanoylcarnitine $(0.5 \mathrm{mM})$ using the recirculating perfusion technique described under Methods. Results are given as means $\pm \mathrm{SEM}$ for five livers in each group.

were initiated while anesthesia was still in effect. As is evident from the data of Fig. 3 the acutely ketotic alloxan diabetic rat is severely ill with marked hypoglycemia and hyperketonemia. The initial plasma glucose concentrations in the three groups of animals at zero time clustered around $1,000 \mathrm{mg} / 100 \mathrm{ml}$, a figure much higher than that found in pancreatectomized diabetic rats (19) but very close to values seen in severe human disease (20). Mean plasma ketone levels were greater than $1,500 \mu \mathrm{mol} / 100 \mathrm{ml}$. When insulin was given alone ketone concentrations decreased from an initial value of $1,760 \mu \mathrm{mol} / 100 \mathrm{ml}$ to $1,255 \mu \mathrm{mol} / 100 \mathrm{ml}$ at $4 \mathrm{~h}$ (Fig. $3 B)$. Over the same time interval $(+)$-decanoylcarnitine, in the absence of insulin, caused a fall in acetoacetate and $\beta$-hydroxybutyrate levels from 1,502 to 262 $\mu \mathrm{mol} / 100 \mathrm{ml}$. When the two agents were given in combination the equivalent $4 \mathrm{~h}$ effect was a decrease in ketones from the zero time value of $1,532 \mu \mathrm{mol} / 100 \mathrm{ml}$ to $69 \mu \mathrm{mol} / 100 \mathrm{ml}$. The relative antiketogenic effect of insulin: (t)-decanoylcarnitine: insulin plus ( + )-decanoylcarnitine was thus $1: 2.5: 3.0$ ( $\Delta$ ketones $-4 \mathrm{~h}, 505$ : $1,240: 1,463 \mu \mathrm{mol} / 100 \mathrm{ml})$. Since the plasma values were normal by four hours in the third group of animals, the accelerating effect of insulin on the reversal of ketosis by $(+)$-decanoylcarnitine is best seen by comparisons made at the $2 \mathrm{~h}$ point. Here the relative antiketogenic ratios were insulin: ( + -decanoylcarnitine: insulin plus $(+)$-decanoylcarnitine, $1: 2.0: 3.5(\Delta$ ketones-2 h, 292:610:991 $\mu \mathrm{mol} / 100 \mathrm{ml}$ ).

The changes in plasma glucose concentrations with the three treatment schedules are shown in Fig. $3 \mathrm{~A}$. 


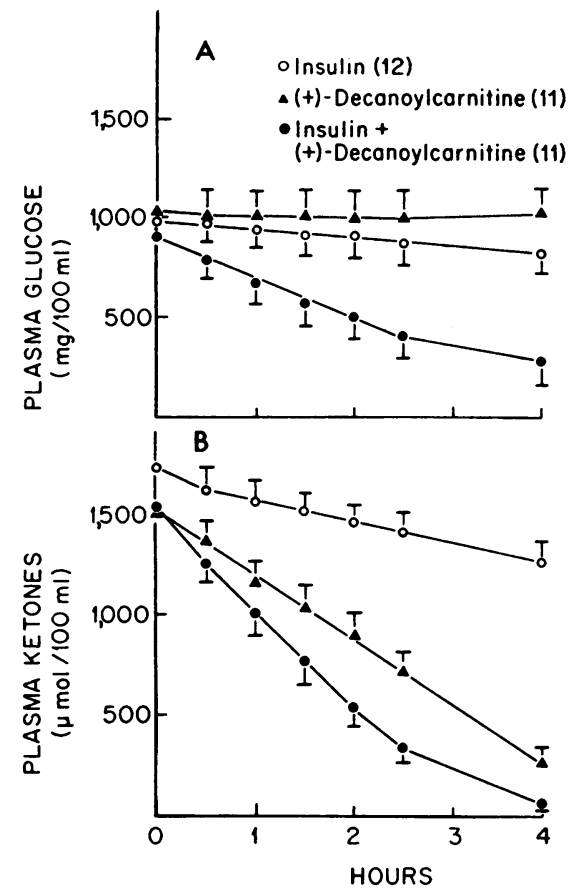

FIgURE 3 The effect of i.v. administration of insulin and $(+)$-decanoylcarnitine in anesthetized diabetic rats. Animals that were still under pentobarbital anesthesia after the placement of arterial and venous catheters were placed in restraining cages and received $100 \mathrm{U}$ of heparin at zero time. After collection of an arterial blood sample $(0.1 \mathrm{ml})$ an infusion of $5 \%$ albumin in $0.9 \%$ sodium chloride, $\mathrm{pH}$ 7.4, containing insulin $(1 \mathrm{U} / \mathrm{ml}),(+)$-decanoylcarnitine $(20 \mathrm{mg} / \mathrm{ml})$ or both agents was given through the venous catheter at a rate of $75 \mu \mathrm{l} / \mathrm{min}$ for $10 \mathrm{~min}$ followed by 10 $\mu \mathrm{l} / \mathrm{min}$ until the $2 \mathrm{~h}$ time point. Further arterial blood samples were collected at the indicated times for analysis of plasma glucose and ketone body concentrations. Values represent means $\pm \mathrm{SEM}$ for the number of animals shown in parentheses.

(+)-Decanoylcarnitine had no effect whatever, the initial value being $1,043 \mathrm{mg} / 100 \mathrm{ml}$ and the $4 \mathrm{~h}$ value being $1,045 \mathrm{mg} / 100 \mathrm{ml}$. Insulin had a hypoglycemic effect, but the total change in $4 \mathrm{~h}$ was quite small, from $976 \mathrm{mg} / 100 \mathrm{ml}$ at zero time to $836 \mathrm{mg} / 100 \mathrm{ml}$ at the end of the experiment. Surprisingly, when (+)-decanoylcarnitine was given with insulin the plasma glucose fell much more rapidly, the initial value of $896 \mathrm{mg} / 100 \mathrm{ml}$ decreasing to $281 \mathrm{mg} / 100 \mathrm{ml}$ at $4 \mathrm{~h}$.

Reversal of diabetic ketoacidosis with (+)-decanoylcarnitine and insulin-nonanesthetized animals. Because of the possibility that continued anesthesia might alter the results of treatment, we next carried out experiments in which catheters were placed under light ether anesthesia. All animals were awake throughout the experiments. Several other differences from the studies shown in Fig. 3 should be noted. First, infusions were carried out for only $10 \mathrm{~min}$ with all agents (i.e., the maintenance infusions given between 10 and 120 min in the experiments of Fig. 3 were omitted). Secondly, no heparin was given to the animals. Thirdly, only control and 2-h samples of blood were obtained in each rat. The results of these experiments are shown in Fig. 4. As indicated in panel $\mathrm{C}$, insulin caused a decrease in acetoacetate plus $\beta$-hydroxybutyrate concentrations from $2,117 \mu \mathrm{mol} / 100$ to $1,869 \mu \mathrm{mol} / 100 \mathrm{ml}(\Delta$, - $248 \mu \mathrm{mol} / 100 \mathrm{ml}$ ). (+)-Decanoylcarnitine as the sole agent of treatment dropped total ketones over the $2 \mathrm{~h}$ period from $1,863 \mu \mathrm{mol} / 100 \mathrm{ml}$ to $1,254 \mu \mathrm{mol} / 100$ $\mathrm{ml}(\Delta,-609 \mu \mathrm{mol} / 100 \mathrm{ml})$. Inclusion of insulin with $(+)$-decanoylcarnitine, exactly as was the case with the first group of animals, caused an accelerated reversal of ketosis, the fall in $2 \mathrm{~h}$ being from 1,754 to $717 \mu \mathrm{mol} /$ $100 \mathrm{ml}(\Delta,-1037 \mu \mathrm{mol} / 100 \mathrm{ml})$. It could thus be concluded that the antiketogenic effects of insulin and $(+)$ decanoylcarnitine were unaffected by anesthesia or heparin administration.

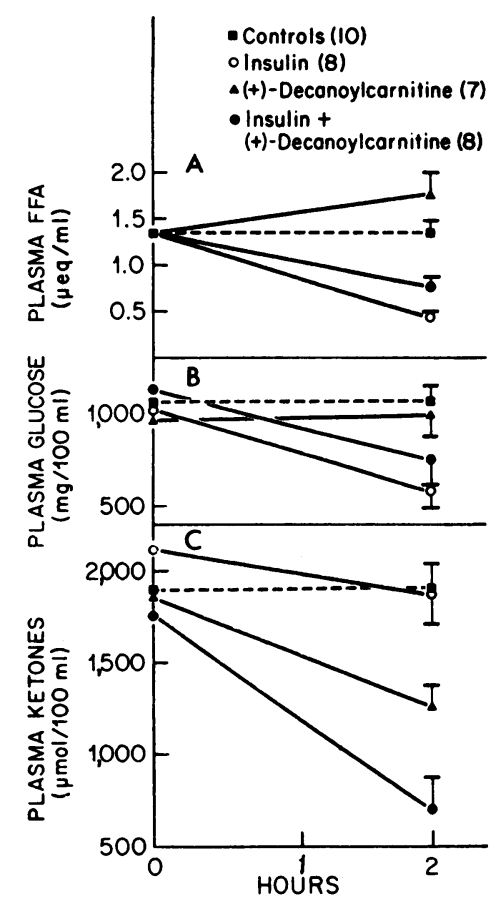

FIgURE 4 The effect of i.v. administration of insulin and $(+)$-decanoylcarnitine in unanesthetized diabetic rats. The experimental conditions differed from those described in Fig. 3 in the following respects: $(a)$ the animals were only lightly anesthetized with ether during the placement of catheters and were fully awake during the experimental period; (b) no heparin was used; (c) the infusion was stopped after the 10 min priming dose had been delivered; $(d)$ at the $2 \mathrm{~h}$ time point the animals were anesthetized with pentobarbital to allow the collection of a large blood sample from the abdominal aorta for analysis of plasma glucose, ketone body, and FFA levels. Values represent means \pm SEM for the number of animals shown in parentheses. 
Such was not the case with the changes in blood glucose, however (Fig. 4B). While control animals and those treated with $(+)$-decanoylcarnitine alone showed no alteration in plasma glucose concentration, confirming the findings shown in Fig. 3, when (+)-decanoylcarnitine was given with insulin, no enhancement of the hormone's hypoglycemic effect was now evident $(\Delta$ glucose, $2 \mathrm{~h}$, insulin, $-423 \mathrm{mg} / 100 \mathrm{ml} ; \Delta$ glucose, $2 \mathrm{~h}$, insulin plus (+)-decanoylcarnitine, $-387 \mathrm{mg} / 100 \mathrm{ml}$ ). The difference in the two experiments appears to be entirely due to a greater hypoglycemic effect of insulin in the awake animals since the $2 \mathrm{~h}$ fall with insulin alone was only $69 \mathrm{mg} / 100 \mathrm{ml}$ in anesthetized rats (Fig. $3 A$ ).

The changes in free fatty acid (FFA) concentrations with treatment are shown in Fig. $4 A$. As expected, insulin caused a decrease in the fatty acid levels, the value falling from 1.34 to $0.42 \mu \mathrm{eq} / \mathrm{ml}$ over the $2 \mathrm{~h}$ period. $(+)$-Decanoylcarnitine appeared to cause an increase in the fatty acid concentration from control values and to blunt the insulin-induced fall.

In the experiments shown in Fig. 5 animals with acute diabetic ketoacidosis were treated with a single

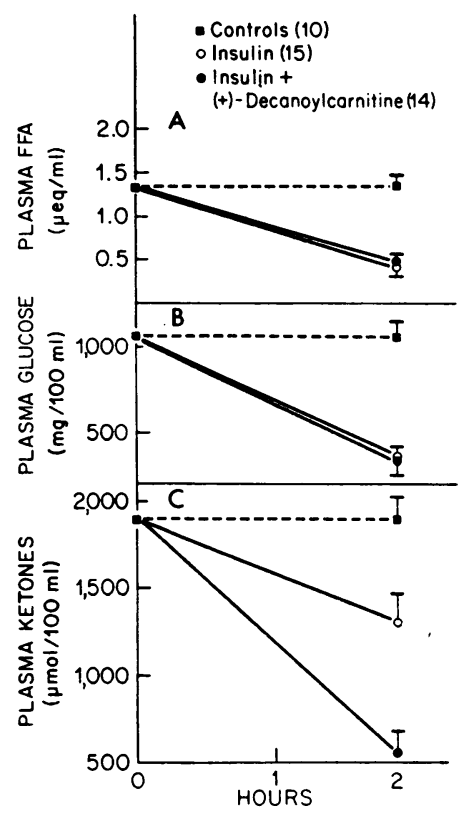

FIGURE 5 The effect of a single injection of insulin with or without $(+)$-decanoylcarnitine in nonanesthetized diabetic rats. Noncatheterized animals received, intravenously over a 2 min period, $0.5 \mathrm{ml}$ of $5 \%$ albumin in $0.9 \%$ sodium chloride containing insulin $(2 \mathrm{U} / \mathrm{ml})$ in the presence or absence of $(+)$-decanoylcarnitine $(20 \mathrm{mg} / \mathrm{ml})$. They were then placed in conventional cages and $2 \mathrm{~h}$ later a large sample of arterial blood was collected for analyses as described in Fig. 4. Values represent means \pm SEM for the number of animals shown in parentheses. Control values have been taken from Fig. 4.

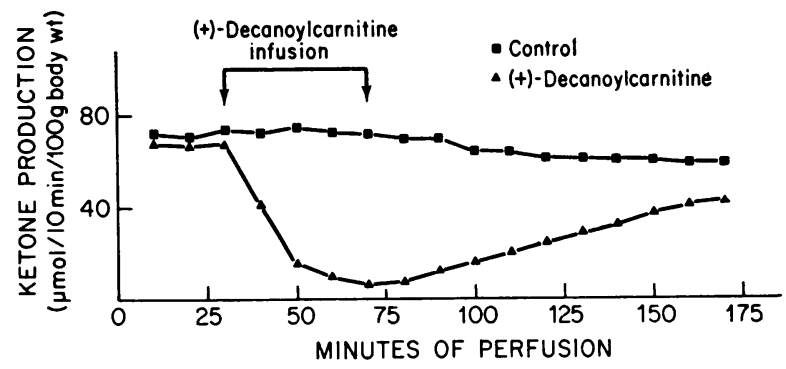

Figure 6 Effect of limited exposure to $(+)$-decanoylcarnitine on ketogenesis in perfused livers from diabetic rats. Livers were perfused with oleic acid $(0.7 \mathrm{mM})$ using the nonrecirculating technique described under Methods. At the $30 \mathrm{~min}$ time point an infusion of $(+)$-decanoylcarnitine

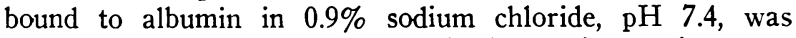
introduced into the perfusion fluid just prior to its entry into the liver such that the concentration of the inhibitor reaching the tissue was $0.5 \mathrm{mM}$. At the 70 min time point the infusion was stopped and the perfusion was continued for a further $100 \mathrm{~min}$. The perfusate leaving the liver was collected in a series of tubes and analyzed for ketone bodies. The control liver was perfused with the albumin solution without (+)-decanoylcarnitine. The figure represents a single experiment, one of four carried out with identical results.

rapid injection of insulin or insulin plus (+)-decanoylcarnitine. They were never anesthetized, had no catheters inserted, and were not placed in restraining cages. $2 \mathrm{~h}$ after treatment they were anesthetized with pentobarbital, and blood was drawn from the aorta. Control values were taken from the animals shown in Fig. 4. Once again the potent antiketogenic effect of $(+)$-decanoylcarnitine over and above that seen with insulin alone was evident. As was the case in the experiments of Fig. 4 the hypoglycemic effect of insulin was not altered by the addition of the carnitine ester. The absolute fall in plasma glucose, assuming that initial values found in control rats were essentially similar to those actually present at zero time in the treated animals, appeared to be considerably greater $(\Delta,-720 \mathrm{mg} / 100$ $\mathrm{ml}$ per $2 \mathrm{~h}$ ) than that seen in the two previous sets of experiments. The insulin-induced fall in free fatty acids was comparable. However, in this case $(+)$-decanoylcarnitine did not reduce the FFA-lowering capacity of insulin. In experiments not shown $(+)$-decanoylcarnitine given alone as a single injection again lowered ketones at a rate faster than that produced by insulin alone but less than that seen when the two agents were combined.

Duration of the (+)-decanoylcarnitine effect in vitro. The studies presented thus far clearly indicated that the effect of (+)-decanoylcarnitine in blocking fatty acid oxidation and ketogenesis was rapid in onset both in vitro and in vivo. Accordingly, we next attempted to get some estimate of the duration of the effect. This 
was done in the isolated rat liver in which a flowthrough perfusion system was established. A series of experiments were carried out in which an infusion of (t)-decanoylcarnitine was given for a short interval and then discontinued. The results of one such experiment, entirely typical, are shown in Fig. $6.10 \mathrm{~min}$ after the addition of $(+)$-decanoylcarnitine the ketone production rate fell from 70 to about $40 \mu \mathrm{mol} / 10 \mathrm{~min}$ per $100 \mathrm{~g}$ body wt. Synthesis of ketones continued to fall and became negligible by $40 \mathrm{~min}$ after the start of the $(+)$-decanoylcarnitine infusion. When the infusion was stopped ketone production began to increase almost immediately and $100 \mathrm{~min}$ later was approaching the control rate. The results indicate, therefore, that the $(+)$-decanoylcarnitine block of fatty acid oxidation is readily reversible on cessation of treatment.

\section{DISCUSSION}

The results reported in this study indicate that $(+)$ decanoylcarnitine, an inhibitor of the long-chain acylcarnitine transferase reaction, has the capacity to interrupt ketone body production in the severely ketotic alloxan diabetic rat and demonstrate that its antiketogenic effect is more powerful than that of insulin itself. Such an observation is entirely consistent with the finding that $(+)$-decanoylcarnitine almost immediately stops ketone body production in the perfused liver of the alloxan diabetic rat (Figs. 2 and 6 ) even though these livers are resistant to the action of antiketogenic agents of the carbohydrate class as well as insulin (3). It is of interest that in vivo the combination of insulin and $(+)$-decanoylcarnitine was more effective than the latter agent alone. The mechanism by which insulin enhances the antiketogenic effect of $(+)$-decanoylcarnitine has not as yet been established. It seems clear that the hormone must ultimately act directly on the liver to reverse ketosis, apart from any effect it has in stopping substrate delivery by blocking free fatty acid release, since elevation of free fatty acid levels in ketotic animals restored to normal by treatment with insulin will not reinitiate ketosis (21). One possibility would be, therefore, that insulin also causes a change in the hepatic acylcarnitine transferase reaction such that an additive response results when both agents are given together ( 6 , 9). Alternatively, while the antiketogenic effect of insulin alone cannot be explained solely by an anti-lipolytic action, its synergistic effect with $(+)$-decanoylcarnitine could be. It is known that long-chain fatty acyl-CoA and ( + -acylcarnitine derivatives interact competitively with acylcarnitine transferase (22). It is thus conceivable that diminished free fatty acid delivery to the liver under the influence of insulin would result in a fall of fatty acyl-CoA levels and an increase in the effectiveness of the $(+)$-decanoylcarnitine block. ${ }^{1}$ Thirdly, the insulin effect might be exerted at the periphery by increasing the rate of ketone body utilization (23). Presumably a fall in free fatty acid delivery to muscle would accelerate the utilization of ketone bodies in that tissue, particularly if coupled with a $(+)$ decanoylcarnitine block in fatty acid oxidation. Preliminary evidence against an insulin effect dependent upon an antilipolytic mechanism has been obtained in experiments (not shown) where free fatty acid concentrations were lowered with nicotinic acid (24) in the presence of $(+)$-decanoylcarnitine. No enhancement of the antiketogenic effect was seen, the $2 \mathrm{~h}$ fall in total ketones being $611 \mu \mathrm{mol} / 100 \mathrm{ml}$ with both compounds given together as opposed to $608 \mu \mathrm{mol} / 100 \mathrm{ml}$ with (+)decanoylcarnitine alone. It should also be noted that the $(+)$-decanoylcarnitine effect could not be reproduced by increasing the levels of insulin up to four times those given in the experiments shown here, a finding reminiscent of the observation in humans that increasing the insulin dosage beyond the usual treatment schedule does not shorten recovery times from diabetic ketoacidosis $(25,26)$.

A second feature of interest in the results was the fact that $(+)$-decanoylcarnitine accelerated the fall in plasma glucose produced by insulin in rats that were anesthetized, but did not do so in awake and moving animals. Hepatic gluconeogenesis has been considered to be stimulated by fatty acid oxidation $(10,11,27)$, at least where rates are very high $(6)$, and $(+)$-decanoylcarnitine has been shown to block this stimulatory effect in the perfused liver $(6,11,27)$. It is conceivable, therefore, that $(+)$-decanoylcarnitine had the same effect in vivo. If the carnitine ester were acting primarily to block hepatic gluconeogenesis, however, it would seem unlikely that the response would not be evident in both anesthetized and unanesthetized animals. For this reason we favor the possibility that the accelerated fall in blood glucose produced by $(+)$-decanoylcarnitine is due to a blockade of fatty acid oxidation in peripheral tissues with the result that glucose would be oxidized more

\footnotetext{
${ }^{1}$ In view of the generalization made earlier that interruption of substrate delivery to the liver should reverse the ketotic state, the question might be raised as to why insulin, which rapidly reduced plasma FFA levels, did not proportionately lower plasma ketone concentrations. The answer undoubtedly lies in the observation that livers from severely ketotic alloxan diabetic rats are engorged with fat $(5,36)$ such that they can sustain high rates of ketogenesis for several hours in the absence of exogenous fatty acids $(3,36)$.

${ }^{2}$ In the experiments shown in Fig. 3 the insulin dosage was $18.5 \mathrm{U} / \mathrm{kg}$ body wt in $2 \mathrm{~h}$. An equivalent schedule in a $70 \mathrm{~kg}$ man would be about $1,300 \mathrm{U}$ in $2 \mathrm{~h}$, a dose some six times greater than that normally administered to a patient in diabetic ketoacidosis (1).
} 
rapidly as the alternative substate (28). A possible interpretation would be that insulin acts essentially to permit (or cause) accelerated transport of glucose across the cell membrane $(29,30)$ in a process that is dependent in part on the intracellular utilization of glucose such that an increased oxidation of glucose would enhance the transport process $(31,32)$. An analogous situation would be muscular exercise, which has long been known to have a hypoglycemic effect in diabetes (33, 34 ), increasing glucose utilization in the presence of insulin presumably by activating glycolysis (35). Indeed, it is attractive to speculate that the failure to demonstrate a synergistic effect of (+)-decanoylcarnitine on the insulin-induced fall in plasma glucose in nonanesthetized animals was due to their increased muscular activity, since even the animals placed in restraining cages were continually moving their limbs. The implication would be that the capacity to increase glucose utilization beyond the level produced by insulin alone is limited and that this limit was reached either by $(+)$-decanoylcarnitine treatment or by increasing muscular exercise.

Despite its potent capacity to block fatty acid oxidation in the liver, and, presumably, in peripheral tissues, no deleterious side effects were seen in rats treated with $(+)$-decanoylcarnitine under the conditions utilized in these experiments, probably because of the plentiful availability of other substrate in the form of glucose and ketone bodies (28). It was also noteworthy that the $(+)$-decanoylcarnitine effect appeared to be readily reversible (Fig. 6). Nevertheless it should be emphasized that the compound is potentially dangerous both from the metabolic standpoint and because it is a surface active agent capable of inducing red blood cell hemolysis in the unbound state. Additional studies of metabolic effects and possible toxicity of $(+)$-decanoylcarnitine are in progress.

We consider these results to be important for the following reasons: (a) They provide additional support for the viewpoint that studies of metabolic regulation in the isolated perfused liver are applicable to the intact animal (6). (b) They are entirely compatible with the postulated critical role of long-chain acylcarnitine transferase in the regulation of hepatic ketogenesis $(1,6-8)$. (c) They raise the possibility that alternative and more effective forms of treatment for diabetic ketoacidosis may one day be available.

\section{ACKNOWLEDGMENTS}

We acknowledge with gratitude the generosity of Dr. Y. Kawashima and the Otsuka Pharmaceutical Factory in supplying the $(+)$-decanoylcarnitine used in these studies. The excellent technical assistance of Ms. Petra Contreras and Martha Cook should also be noted.

This investigation was supported by U. S. Public Health Service Grant CA-08269.

\section{REFERENCES}

1. McGarry, J. D., and D. W. Foster. 1972. Regulation of ketogenesis and clinical aspects of the ketotic state. Metab. (Clin. Exp.). 21: 471.

2. McGarry, J. D., M. J. Guest, and D. W. Foster. 1970. Ketone body metabolism in the ketosis of starvation and alloxan diabetes. J. Biol. Chem. 245: 4382.

3. McGarry, J. D., and D. W. Foster. 1971. The regulation of ketogenesis from octanoic acid. The role of the tricarboxylic acid cycle and fatty acid synthesis. J. Biol. Chem. 246: 1149.

4. McGarry, J. D., and D. W. Foster. 1971. The regulation of ketogenesis from oleic acid and the influence of antiketogenic agents. J. Biol. Chem. 246: 6247.

5. Meier, J. M., J. D. McGarry, G. R. Faloona, R. H. Unger, and D. W. Foster. 1972. Studies of the development of diabetic ketosis in the rat. J. Lipid Res. 13:228.

6. McGarry, J. D., J. M. Meier, and D. W. Foster. 1973. The effects of starvation and refeeding on carbohydrate and lipid metabolism in vivo and in the perfused rat liver. The relationship between fatty acid oxidation and esterification in the regulation of ketogenesis. J. Biol. Chem. 243: 270 .

7. Fritz, I. B. 1967 An hypothesis concerning the role of carnitine in the control of interrelations between fatty acid and carbohydrate metabolism. Perspect. Biol. Med. 10: 643 .

8. Augenfeld, J., and I. B Fritz. 1970. Carnitine palmitoyltransferase activity and fatty acid oxidation by livers from fetal and neonatal rats. Can. J. Biochem. 48: 288.

9. Norum, R. N. 1965. Activation of palmityl-CoA : carnitine palmityltransferase in livers from fasted, fat-fed or diabetic rats. Biochem. Biophys. Acta. 98: 652 .

10. Delisle, G., and I. B Fritz. 1967. Interrelations between hepatic fatty acid oxidation and gluconeogenesis: a possible regulatory role of carnitine palmityltransferase. Proc. Natl. Acad.Sci. U. S. A. 58: 790.

11. Williamson, J. R., E. T. Browning, R. Scholz, R. A. Kreisberg, and I. B. Fritz. 1968. Inhibition of fatty acid stimulation of gluconeogenesis by $(+)$-decanoylcarnitine in perfused rat liver. Diabetes. 17: 194.

12. Williamson, J. R., E. T. Browning, R. G. Thurman, and R. Scholz. 1969. Inhibition of glucagon effects in perfused rat liver by $(+)$-decanoylcarnitine. J. Biol. Chem. 244: 5055 .

13. Foster, D. W 1967. Studies in the ketosis of fasting. $J$. Clin. Invest. 46: 1283.

14. Saifer, A., and S. Gerstenfeld. 1958. The photometric microdetermination of blood glucose with glucose oxidase. J Lab. Clin. Med. 51 : 448.

15. Dole, V. P. 1956. A relation between non-esterified fatty acids in plasma and the metabolism of glucose. J. Clin. Invest. $35: 150$.

16. Trout, D. L., E. H. Estes, Jr., and S. J. Friedberg. 1960. Titration of free fatty acids of plasma: a study of current methods and a new modification. J. Lipid Res. $1: 199$.

17. Exton, J. H., and C. R. Park. 1967. Control of gluconeogenesis in liver. I. General features of gluconeogenesis in the perfused livers of rats. J. Biol. Chem. 242: 2622.

18. Exton, J. H., J. C. Corbin, and S. C. Harper. 1972. Control of gluconeogenesis in liver. V. Effects of fasting, diabetes, and glucagon on lactate and endogenous metabolism in the perfused rat liver. J. Biol. Chem. 247:4996. 
19. Chernick, S. S., and R. O Scow. 1959. Early effects of total pancreatectomy on fat metabolism in the rat. $\mathrm{Am}$. J. Physiol. 196: 125.

20. Beigelman, P. M 1971. Severe diabetic ketoacidosis (diabetic coma). 482 episodes in 257 patients; experience of three years. Diabetes. 20: 490.

21. Bieberdorf, F. A., S. S. Chernick, and R. O. Scow. 1970. Effect of insulin and acute diabetes on plasma FFA and ketone bodies in the fasting rat. J. Clin. Invest. 49 : 1685 .

22. Fritz, I. B., and N. R. Marquis. 1965. The role of acylcarnitine esters and carnitine palmityltransferase in the transport of fatty acyl groups across mitochondrial membranes. Proc. Natl. Acad. Sci. U. S. A. 54: 1226.

23. Balasse, E. O., and R. J. Havel. 1970 Turnover rate and oxidation of ketone bodies in normal and diabetic dogs Diabetologia. $6: 36$.

24. Carlson, L. A. 1969. Antilipolysis as a tool in the study of clinical and experimental diabetes. Diabetologia. 5: 361.

25. Smith, K., and H. E. Martin. 1954. Response of diabetic coma to various insulin dosages. Diabetes. 3: 287.

26. Shaw, C. E., G. E. Horwitz, M. Schmukler, S. H. Brager, and S. P. Bessman. 1962. A clinical and laboratory study of insulin dosage in diabetic acidosis: comparison with small and large doses. Diabetes. 11: 23.

27. Williamson, J. R. 1967. Effects of fatty acids, glucagon and anti-insulin serum on the control of gluconeogenesis and ketogenesis in rat liver. Adv. Enzyme Regul. 5:229.

28. Randle, P. J., P. B. Garland, C. N. Hales, and E. A. Newsholme. 1963. The glucose fatty-acid cycle. Its role in insulin sensitivity and the metabolic disturbances of diabetes mellitus. Lancet. $1: 785$.

29. Park, C. R., D. Reinwein, M. J. Henderson, E. Cadenas, and H. E. Morgan. 1959. The action of insulin on the transport of glucose through the cell membrane. Am. J. Med. $26: 674$.

30. Cahill, G. F., Jr. 1971. Physiology of insulin in man. Diabetes. 20: 785 .

31. Morgan, H. E., M. J. Henderson, D. M. Regen, and C. R. Park. 1961. Regulation of glucose uptake in muscle. I. The effects of insulin and anoxia on glucose transport and phosphorylation in the isolated perfused heart of normal rats. J. Biol. Chem. 236: 253.

32. Özand, P., H. T. Narahara, and C. F. Cori. 1962. Studies of tissue permeability. VIII. The effect of anaerobiosis on glucose uptake in frog sartorius muscle. J. Biol. Chem. 237: 3037.

33. Lawrence, R. D. 1926. The effect of exercise on insulin action in diabetes. Br. Med.J. $1: 648$.

34. Klachko, D. M., T. H. Lie, E. J. Cunningham, G. R. Chase, and T. W. Burns. 1972. Blood glucose levels during walking in normal and diabetic subjects. Diabetes. $21: 89$.

35. Huckabee, W. E. 1958. Relationships of pyruvate and lactate during anaerobic metabolism. II. Exercise and formation of $\mathrm{O}_{2}$-debt. J. Clin. Invest. 37: 255.

36. Van Harken, D. R, C. W. Dixon, and M. Heimberg. 1969. Hepatic lipid metabolism in experimental diabetes. V. The effect of concentration of oleate on metabolism of triglycerides and on ketogenesis. J. Biol. Chem. 244: 2278. 